\title{
Atuação do enfermeiro durante o pós-parto de pacientes com transtornos mentais
}

\section{puerperais}

\author{
Nurse's role during the postpartum period of patients with puerperal mental disorders \\ Papel de la enfermera durante el puerperio de pacientes con trastornos mentales puerperales
}

Recebido: 26/08/2021 | Revisado: 30/08/2021 | Aceito: 03/09/2021 | Publicado: 04/09/2021

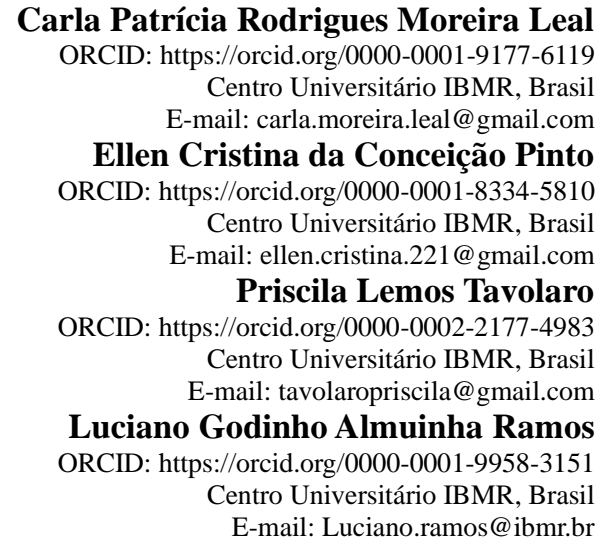

\section{Resumo}

O presente estudo versa sobre a atuação do enfermeiro durante o pós-parto, com pacientes com transtornos mentais puerperais. Tem como objetivo destacar a importância da atuação do enfermeiro durante o pós-parto de mulheres que apresentam transtornos mentais no puerpério. Foi efetuada uma revisão integrativa com a seguinte questão norteadora: Qual a importância da atuação do enfermeiro durante o pós-parto, frente a pacientes com transtornos mentais puerperais? A busca das publicações foi realizada nas bases de dados Medline, Lilacs, BDENF e Scielo. De acordo com os critérios de inclusão, foram selecionados dez artigos, sete publicados na língua portuguesa e três publicados na língua inglesa, com texto disponível na íntegra, entre os meses de março a maio de 2021, utilizando-se os seguintes descritores: "Transtornos mentais", “depressão pós-parto", "gravidez”, "papel do enfermeiro". Para a discussão foi realizada a categorização dos trabalhos por similaridade de conteúdo, sendo construídas três categorias de discussão para a análise: "Depressão pós-parto - Uma realidade solitária no cuidado com a puérpera"; "Papel do enfermeiro com pacientes com transtornos mentais puerperais - O início de uma longa jornada" e "Estratégias e protocolos na assistência às puérperas com transtornos mentais puerperais". Conclui-se que há a necessidade de que o enfermeiro tenha conhecimento sobre transtornos mentais puerperais para, assim, acompanhar a mulher de forma ampla, oferecendo-lhe uma assistência integral, a partir do período gestacional até o puerpério, reduzindo o risco de agravos futuros à sua saúde e à de seu bebê.

Palavras-chave: Transtornos mentais; Depressão pós-parto; Gravidez; Papel do enfermeiro.

\begin{abstract}
The present study is about the role of nurses during the postpartum with patients with puerperal mental disorders. The main objective is the importance of the role of nurses during the postpartum period for women with mental disorders in the puerperium. An integrative review was carried out with the following guiding question: "What is the importance of the nurse's performance during the postpartum period, in front of patients with puerperal mental disorders?" The search for publications was performed in Medline, Lilacs, BDENF and Scielo databases. According to the inclusion criteria, ten articles were selected, seven published in the Portuguese language and three published in the English language, with full text available, from March to May 2021, using the following descriptors: "disorders mental disorders", "postpartum depression", "pregnancy", "role of the nurse". For the discussion, the works were categorized by content similarity, and three discussion categories were built for analysis: "Postpartum depression - A lonely reality in the care of the puerperal woman; "The role of nurses with patients with puerperal mental disorders - The beginning of a long journey" and "Strategies and protocols in assisting mothers with puerperal mental disorders". We conclude that there is a need for the nurse to have knowledge about puerperal mental disorders in order to, thus, accompany the woman in a broad way, offering her comprehensive assistance, from the gestational period until the puerperium, reducing the risk of future injuries your health to that of your baby.
\end{abstract}

Keywords: Mental disorders; Postpartum depression; Pregnancy; Nurse's role. 


\begin{abstract}
Resumen
Este estudio aborda el papel de las enfermeras durante el posparto, con pacientes con trastornos mentales posparto. Tiene como objetivo resaltar la importancia del papel de las enfermeras durante el puerperio para las mujeres con trastornos mentales en el puerperio. Se realizó una revisión integradora con la siguiente pregunta orientadora: ¿Cuál es la importancia del rol de la enfermera durante el puerperio, en relación a las pacientes con trastornos mentales puerperales? La búsqueda de publicaciones se realizó en las bases de datos Medline, Lilacs, BDENF y Scielo. Según los criterios de inclusión, se seleccionaron diez artículos, siete publicados en portugués y tres publicados en inglés, con el texto disponible en su totalidad, entre los meses de marzo y mayo de 2021, utilizando los siguientes descriptores: "Trastornos mentales", "depresión posparto ", “ Embarazo ”, " el papel de la enfermera ”. Para la discusión, los trabajos fueron categorizados por similitud de contenido y se construyeron tres categorías de discusión para su análisis: "Depresión posparto - Una realidad solitaria en el cuidado de la puérpera"; "Role de la enfermera con pacientes con trastornos mentales puerperales - El inicio de un largo camino" y "Estrategias y protocolos en la atención de madres con trastornos mentales puerperales". Se concluye que existe la necesidad de que las enfermeras tengan conocimientos sobre los trastornos mentales puerperales para poder monitorear a las mujeres de manera integral, brindándoles una atención integral, desde el período gestacional hasta el puerperio, reduciendo el riesgo de futuras lesiones en su salud y que de su bebé.
\end{abstract}

Palabras clave: Trastornos mentales; Depresión posparto; Embarazo; Role de la enfermera.

\title{
1. Introdução
}

Receber a confirmação de uma gravidez é para a mulher, na maioria das vezes, um momento de realização, de concretização de um sonho. No entanto, os nove meses de gestação e principalmente o período compreendido entre a primeira hora pós-parto e o quadragésimo segundo dia (período puerperal ou puerpério), são acompanhados de muitas mudanças envolvendo a recente mãe (puérpera), mas também todo o seu círculo de relações. Um número substancial de mulheres, vivencia durante este período, sintomas perturbadores que podem fazer desta experiência a pior da sua vida (Macedo et al., 2014).

Com o nascimento de um bebê, é comumente se afirmar que "nasce também uma mãe", uma vez que a puérpera ao receber o seu filho nos braços, recebe também um aumento brusco de responsabilidade por se tornar, a partir daquele momento, referência de um ser vivo totalmente dependente de seus cuidados e assistência, além de se deparar com a necessidade de uma reorganização psicossocial e de adaptação a um novo papel e identidade. Estas mudanças, juntamente com as desordens físicas e hormonais próprias do puerpério, podem gerar desânimo, insegurança, reforçando a condição de vulnerabilidade natural e inerente a esta fase da sua vida, mas que tende a cessar naturalmente ao longo dos meses, com o fortalecimento e confiança na relação do binômio mãe-bebê e com o apoio do parceiro, de familiares e amigos próximos. Ou não, estas alterações podem frustrar o sentimento inicial de felicidade da recém-mãe, o que pode culminar em alguma doença intercorrente da gravidez e do puerpério, como algum tipo de transtorno mental (Montenegro \& Filho, 2017).

De acordo com a definição presente no Manual Diagnóstico e Estatístico de Transtornos Mentais (American Psychiatric Association, 2014), o transtorno mental é uma síndrome caracterizada por perturbação clinicamente significativa na cognição, na regulação emocional ou no comportamento de um indivíduo que reflete uma disfunção nos processos psicológicos, biológicos ou de desenvolvimento subjacentes ao funcionamento mental. Esta psicopatologia, está frequentemente associada a sofrimento ou incapacidade significativa que afeta atividades sociais, profissionais ou outras atividades importantes.

Segundo a Organização Mundial da Saúde (2010), os transtornos mentais constituem um agravante à saúde pública, mais de 400 milhões de pessoas sofrem de algum transtorno mental. E nesse contexto, estudos atuais demonstram que em relação aos homens, as mulheres são mais suscetíveis ao desenvolvimento de doenças mentais, embora esta diferença esteja cada vez mais próxima, principalmente no que concerne a transtornos depressivos (OPAS, 2017). Onde a gestação e o puerpério podem surgir como fatores predisponentes do desenvolvimento e exacerbação de problemas de saúde mental (Macedo et al., 2014). 
Entre os transtornos mentais que podem surgir no decorrer da gravidez e após o nascimento do bebê, destaca-se a depressão pós-parto (DPP). A DPP se diferencia de outros tipos de depressão, ela se insere em uma trilogia de distúrbios da psiquiatria perinatal, classicamente caracterizada por três entidades distintas: O "Baby Blues", tido também como "Blues" puerperal ou tristeza puerperal, as psicoses puerperais e as depressões pós-parto; e se destaca como protagonista de diversos trabalhos científicos que abordam esta temática. Esta doença é capaz de gerar alterações somáticas e cognitivas, no pós-parto é uma condição em que a mulher se encontra num estado de profunda tristeza, logo após a dar à luz ao seu filho, não existe uma causa especifica, mas estudos mostram que diversos fatores podem desencadear o aparecimento da depressão. A violência obstétrica, violência por parte do parceiro, a gravidez de alto risco, a descoberta de alterações genéticas no feto, a gravidez na adolescência e até mesmo o baixo nível socioeconômico, são fatores de predisposição.

Deste modo, acredita-se que o papel do enfermeiro seja de suma importância para diminuir consideravelmente a ocorrência destas doenças na mulher, pois ele é o profissional que mais interage com a gestante desde o pré-natal até ao nascimento do bebê. Através da consulta de enfermagem, este profissional pode identificar situações de risco e intercorrências no ciclo gravídico-puerperal, que podem desencadear algum tipo de transtorno mental futuro. Ele poderá, desta forma, intervir precocemente anulando o surgimento destas patologias ou evitando o seu avanço para formas mais graves, com uma abordagem humanizada, focada na mulher como protagonista deste grande evento e assim, proporcionar uma assistência adequada e melhor qualidade de vida, não somente para a esta, mas para todos os que fazem parte do seu círculo de relações e em particular, para o recém-nascido (Brasil, 2000).

Assim, o presente trabalho teve como objetivo destacar a importância do enfermeiro durante o pós-parto, frente a pacientes com transtornos psicológicos puerperais.

\section{Metodologia}

Trata-se de uma pesquisa do tipo revisão integrativa com a seguinte questão norteadora: Qual a importância da atuação do enfermeiro durante o pós-parto, frente a pacientes com transtornos mentais puerperais? Este método tem como finalidade sintetizar os resultados obtidos em pesquisas realizadas sobre um mesmo tema ou questão, de forma ordenada e abrangente, fornecendo informações mais amplas sobre um assunto ou problema da área da saúde. Constituindo, assim, um corpo de conhecimento científico mais acessível, pois possibilita ao profissional da área o alcance de diversas pesquisas realizadas, em um único estudo. Além de servir como meio de facilitar o preenchimento de lacunas do conhecimento, através do estimulo para a realização de novos estudos (Ercole et.al, 2014).

Para a elaboração da revisão integrativa foram percorridas as seguintes etapas: definição do objetivo; formulação da questão norteadora: "Qual a importância da atuação do enfermeiro durante o pós-parto, frente a pacientes com transtornos mentais puerperais?"; busca das referências, por meio da Biblioteca Virtual em Saúde (BVS), que faz procura simultânea nas principais bases de dados de amplitude nacional e internacional, sendo selecionadas por este filtro, as bases: Lilacs (Literatura Latino-Americana e do Caribe em Ciências da Saúde), BDENF (Base de dados em Enfermagem) e MEDLINE (Sistema Online de Busca e Análise de Literatura Médica), e também através da base de dados SciELO (Scientific Electronic Library Online), no período de março a abril de 2021, utilizando os descritores: “transtornos mentais”, “depressão pós-parto”, “gravidez” e "papel do enfermeiro", indexados nos Descritores em Ciências da Saúde (DECS). Optou-se pela utilização do operador booleano "AND" entre os descritores selecionados, em detrimento do operador booleano "OR", para se ampliar o número de resultados possíveis, definindo-se como estratégia de procura: "transtornos mentais" AND "depressão pós-parto" AND "gravidez" AND "papel do enfermeiro", da qual resultaram 3 artigos (todos da base de dados Medline). Devido ao número reduzido de artigos mais 5 passos estratégicos de procuram foram estabelecidos: $1^{\circ}$ passo: “depressão pós-parto" AND 
"gravidez" AND "papel do enfermeiro", 8 artigos foram encontrados (nas bases de dados Medline, Lilacs, BDENF e Scielo); $2^{\circ}$ passo: "transtornos mentais" AND "gravidez" AND "papel do enfermeiro", 13 resultados de pesquisa (Base de dados: Scielo e Medline); $3^{\circ}$ passo: "gravidez" AND "papel do enfermeiro", 197 documentos encontrados para a pesquisa (base de dado: Medline, Lilacs e BDENF) ; $4^{\circ}$ passo: "depressão pós-parto" AND "papel do enfermeiro", 47 artigos disponíveis para a pesquisa (Scielo, Medline, Lilacs e BDENF); $5^{\circ}$ passo: "transtornos mentais" AND "depressão pós-parto" AND "papel do enfermeiro, com 3 resultados apenas para esta pesquisa (base de dados Medline). A presença constante do descritor "papel do enfermeiro" em todas as etapas de procura foi enfocada por ser primordial da questão norteadora, juntamente com o descritor "depressão pós-parto". No entanto também foi feita a busca isolada do descritor "gravidez" em conjunto com o descritor "papel do enfermeiro" e destes dois com o descritor "transtornos mentais", visto o contexto gravidez funcionar como desencadeador da depressão pós-parto e neste âmbito surgirem documentos relevantes para o trabalho. O mesmo não foi feito com o descritor "transtornos mentais", pois este não representa uma situação de desenvolvimento da DPP, ele pode surgir como consequência da gestação, do puerpério, e da qual faz parte a DPP e outros transtornos puerperais. Entendeu-se que a sua pesquisa isolada traria uma variedade de conteúdo com pouca expressão para o estudo.

Em seguida foi efetuada a procura pelos artigos, de forma on-line, onde os critérios de inclusão foram: responder à questão norteadora, ter publicação em língua portuguesa, inglesa, espanhola ou francesa, conter texto completo e com disponibilidade on-line na íntegra, gratuito, com publicação realizada no máximo há 5 anos (2016 a 2021), pois pensou-se que um corte temporal superior, tornasse o estudo desatualizado; e os de exclusão foram: artigos pagos, artigos de revisão, resumo de artigos, capítulos de livros, estudos, artigos que não respondessem à questão norteadora, artigos disponíveis em sites não reconhecidos pela comunidade científica e artigos publicados fora do período citado.

A extração das informações dos artigos, em um primeiro momento, foi feita através de uma leitura dos títulos e de uma leitura dos seus resumos, e em um segundo momento, pela leitura integral desses artigos, objetivando-se a eleição daqueles que realmente apresentassem aspetos relevantes para o estudo, procedida pela elaboração de um banco de dados com as informações pertinentes e do preenchimento do quadro sinóptico.

Em relação à avaliação dos artigos, esta foi realizada através de uma leitura crítica, conforme é possível observar na figura 1, que evidencia a seleção total dos artigos encontrados nas bases de dados e após a aplicação dos critérios de inclusão e exclusão, até à eleição do número final de artigos. Para finalizar a revisão integrativa, houve a criação de categorias de discussão, com análise dos tópicos que se sobressaíram na leitura. 
Figura 1 - Esquematização da seleção total dos artigos elegíveis para o estudo.

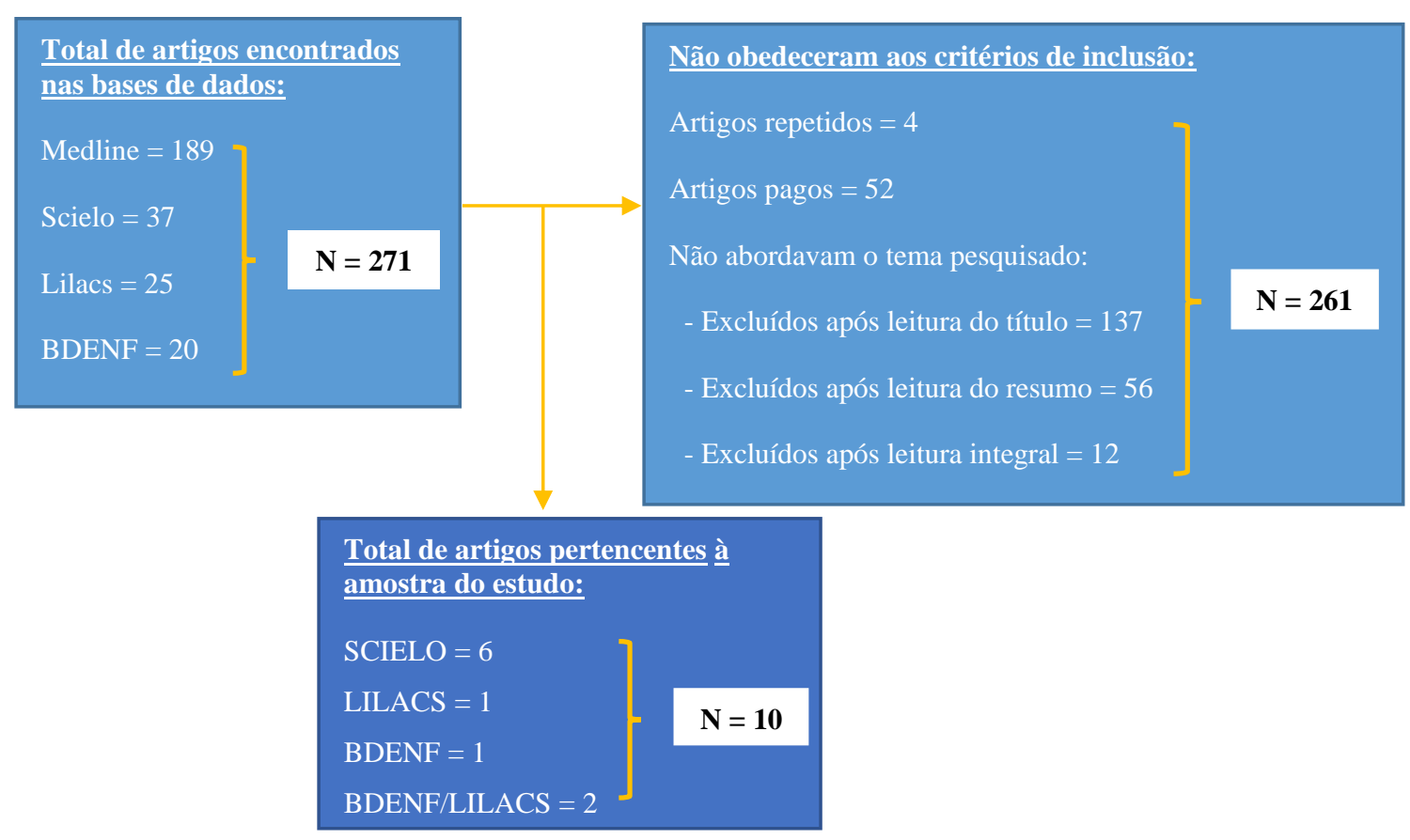

Fonte: Elaborado pelos autores (2021)

\section{Resultados e Discussão}

Conforme a informação compilada no Quadro 1, distribuída entre os seguintes tópicos: título, autor do artigo, ano de publicação, base de dados, objetivo e método, podemos observar que: esta revisão teve a sua amostra composta por 10 artigos, sendo 7 escritos na língua portuguesa e 3 na língua inglesa; em relação à base de dados de origem, 6 se encontravam na base de dados SCIELO, 1 na base de dados BDENF, 1 na base de dados LILACS e 2 se duplicaram na base de dados BDENF e LILACS.

Quanto ao ano de publicação, os artigos elegíveis para esta revisão integrativa, seguiram o recorte temporal previamente estabelecido no critério de inclusão - os últimos 5 anos - onde foram obtidos: 3 (30\%) artigos do ano de 2020, 2 (20\%) artigo do ano de 2019, 2 artigos (20\%) do ano de 2018, 2 artigo (20\%)) do ano de 2017 e 1 artigo do ano de 2016 (10\%). Nesta busca não foram encontrados artigos publicados durante o primeiro semestre do ano vigente (2021).

No que diz respeito à metodologia desenvolvida nos estudos escolhidos, tivemos: 1 (10\%) artigo que apresentou abordagem descritiva qualitativa, 1 (10\%) com abordagem descritiva quantitativa, 1 (10\%) estudo com abordagem exploratória, 1 (10\%) com abordagem transversal, 1 (10\%) com abordagem correlacional e 5 (50\%) seguiram uma abordagem de levantamento bibliográfico. Quanto ao tipo de pesquisa, predominaram os estudos de revisão integrativa. 
Quadro 1 - Distribuição dos artigos elegíveis após leitura integral do conteúdo.

\begin{tabular}{|c|c|c|c|c|c|}
\hline Título do artigo & Autor & $\begin{array}{c}\text { Ano de } \\
\text { publicaçãa }\end{array}$ & $\begin{array}{c}\text { Base de } \\
\text { dados }\end{array}$ & Objetivo & Método \\
\hline $\begin{array}{l}\text { A1 - Content and format } \\
\text { preferences of a depression } \\
\text { prevention program: A } \\
\text { study in perinatal women }\end{array}$ & $\begin{array}{l}\text { Osma, Suso- } \\
\text { Ribera, } \\
\text { Martínez- } \\
\text { Borba e } \\
\text { Barrera }\end{array}$ & 2020 & SCIELO & $\begin{array}{l}\text { Explorar diferenças étnicas nas } \\
\text { preferências de conteúdo e } \\
\text { formato para um programa } \\
\text { preventivo de depressão } \\
\text { perinatal }\end{array}$ & Exploratório \\
\hline $\begin{array}{l}\text { A2 - Estratégias de } \\
\text { Enfermagem na Depressão } \\
\text { Pós-Parto. }\end{array}$ & $\begin{array}{l}\text { Viana } \\
\text { MDZS, } \\
\text { Fettermann } \\
\text { FA, Cesar } \\
\text { MBN }\end{array}$ & 2020 & $\begin{array}{l}\text { BDENF / } \\
\text { LILACS }\end{array}$ & $\begin{array}{l}\text { Identificar na literatura as } \\
\text { estratégias utilizadas pelos (as) } \\
\text { enfermeiros (as) na prevenção da } \\
\text { depressão pós-parto. }\end{array}$ & $\begin{array}{l}\text { Levantamento } \\
\text { bibliográfioc/Revi } \\
\text { são integrativa }\end{array}$ \\
\hline $\begin{array}{l}\text { A3 - Intervenções do } \\
\text { Enfermeiro na atenção e } \\
\text { prevenção da depressão } \\
\text { puerperal. }\end{array}$ & $\begin{array}{l}\text { Silva JF da, } \\
\text { Nascimento } \\
\text { MFC, Silva } \\
\text { AF da, } \\
\text { Oliveira OS } \\
\text { de, Santos } \\
\text { EA, Ribeiro } \\
\text { FMSS }\end{array}$ & 2020 & BDENF & $\begin{array}{l}\text { Identificar na produção científica } \\
\text { sobre as ações/intervenções que } \\
\text { podem ser desenvolvidas pelo } \\
\text { enfermeiro na atenção e } \\
\text { prevenção de danos da depressão } \\
\text { puerperal. }\end{array}$ & $\begin{array}{l}\text { Levantamento } \\
\text { bibliográfioc/Revi } \\
\text { são integrativa }\end{array}$ \\
\hline $\begin{array}{l}\text { A4 - Depressão pós-parto: } \\
\text { Identificação de Sinais, } \\
\text { sintomas e fatores } \\
\text { associados em maternidade } \\
\text { de referência em Manaus. }\end{array}$ & $\begin{array}{l}\text { Aloise, } \\
\text { Ferreira \& } \\
\text { Lima }\end{array}$ & 2019 & $\begin{array}{l}\text { BDENF / } \\
\text { LILACS }\end{array}$ & $\begin{array}{l}\text { Identificar sinais e sintomas de } \\
\text { Depressão Pós-Parto (DPP) e } \\
\text { fatores associados em mulheres } \\
\text { no puerpério mediato, entre } 48 \mathrm{~h} \\
\text { e } 72 \mathrm{~h} \text {. }\end{array}$ & $\begin{array}{l}\text { Estudo descritivo } \\
\text { quantitativo }\end{array}$ \\
\hline $\begin{array}{l}\text { A5 - Postpartum } \\
\text { Depression: } \\
\text { Epidemiological Clinical } \\
\text { Profile of Patients Attended } \\
\text { In a Reference Public } \\
\text { Maternity in Salvador-BA }\end{array}$ & $\begin{array}{l}\text { Araújo, } \\
\text { Aquino, } \\
\text { Fagundes e } \\
\text { Santos }\end{array}$ & 2019 & LILACS & $\begin{array}{l}\text { Avaliar o perfil clínico } \\
\text { epidemiológico de mulheres com } \\
\text { suspeita de Depressão } \\
\text { Pós-Parto em uma maternidade } \\
\text { pública de referência de } \\
\text { Salvador, no estado da BA }\end{array}$ & Estudo transversal \\
\hline $\begin{array}{l}\text { A6 -.Depressão pós-parto: } \\
\text { um olhar criterioso da } \\
\text { Equipe de Enfermagem }\end{array}$ & Souza et. al & 2018 & SCIELO & $\begin{array}{l}\text { Conhecer a assistência de } \\
\text { enfermagem designada para } \\
\text { mulheres com quadro de } \\
\text { depressão pós-parto, bem como, } \\
\text { sensibilizar profissionais e } \\
\text { acadêmicos de áreas afins para } \\
\text { aspectos relacionados ao } \\
\text { diagnóstico, percepção familiar e } \\
\text { tratamento da DPP em } \\
\text { puérperas. }\end{array}$ & $\begin{array}{l}\text { Levantamento } \\
\text { bibliográfioc/Revi } \\
\text { são integrativa. }\end{array}$ \\
\hline $\begin{array}{l}\text { A7 - Ações do programa de } \\
\text { puerpério na atenção } \\
\text { primária: uma revisão } \\
\text { integrativa. }\end{array}$ & $\begin{array}{l}\text { Baratieri, } \\
\text { Tatiane; } \\
\text { Natal, Sonia }\end{array}$ & 2018 & SCIELO & $\begin{array}{l}\text { Sistematizar o conhecimento } \\
\text { produzido sobre as ações de } \\
\text { programas de atenção pós-parto } \\
\text { no âmbito da APS, tanto em } \\
\text { nível nacional, como } \\
\text { internacional. }\end{array}$ & $\begin{array}{l}\text { Levantamento } \\
\text { bibliográfioc/Revi } \\
\text { são integrativa }\end{array}$ \\
\hline $\begin{array}{l}\text { A8 - O enfermeiro no pré- } \\
\text { natal de alto Risco: papel } \\
\text { profissional }\end{array}$ & Junior et.al & 2017 & SCIELO & $\begin{array}{l}\text { Conhecer o papel do enfermeiro } \\
\text { no atendimento ao pré-natal de } \\
\text { alto risco realizado na atenção } \\
\text { secundária. }\end{array}$ & $\begin{array}{l}\text { Estudo qualitativo } \\
\text { descritivo. }\end{array}$ \\
\hline
\end{tabular}




\begin{tabular}{|c|c|c|c|c|c|}
\hline $\begin{array}{l}\text { A9 - Stressful Life Events } \\
\text { and Women's Mental } \\
\text { Health During Pregnancy } \\
\text { and Postpartum Period }\end{array}$ & $\begin{array}{l}\text { Alvarenga \& } \\
\text { Frizzo }\end{array}$ & 2017 & SCIELO & $\begin{array}{l}\text { Investigar as relações entre } \\
\text { variáveis sociodemográficas, } \\
\text { estressores durante a gestação e } \\
\text { a saúde mental da mulher na } \\
\text { gestação e no puerpério }\end{array}$ & $\begin{array}{l}\text { Estudo } \\
\text { correlacional } \\
\text { observacional }\end{array}$ \\
\hline $\begin{array}{l}\text { A10 - Ações de Educação } \\
\text { em Saúde na Atenção } \\
\text { Primária a Gestantes e } \\
\text { Puérperas: Revisão } \\
\text { Integrativa. }\end{array}$ & $\begin{array}{l}\text { Camillo BS, } \\
\text { Nietsche EA, } \\
\text { Salbego C et } \\
\text { al. }\end{array}$ & 2016 & SCIELO & $\begin{array}{l}\text { Identificar evidências acerca das } \\
\text { percepções de gestantes e } \\
\text { puérperas sobre as ações de } \\
\text { educação em saúde na atenção } \\
\text { primária }\end{array}$ & $\begin{array}{l}\text { Levantamento } \\
\text { bibliográfioc/Revi } \\
\text { são integrativa }\end{array}$ \\
\hline
\end{tabular}

Fonte: Elaborado pelos autores (2021)

Para a análise crítica dos estudos selecionados foi realizada a categorização dos trabalhos por similaridade de conteúdo, tendo-se construído 3 categorias de discussão: "Depressão pós-parto - Uma realidade solitária no cuidado com a puérpera"; "Papel do enfermeiro com pacientes com transtornos mentais puerperais - O início de uma longa jornada" e "Estratégias e protocolos na assistência às puérperas com transtornos mentais puerperais".

\subsection{Depressão pós-parto - uma realidade solitária no cuidado com a puérpera}

No que concerne à análise dos artigos que compuseram a amostra deste estudo, verificou-se que existe desconhecimento em relação aos transtornos mentais puerperais que podem acometer as grávidas e as recém-mães. Geralmente a depressão pós-parto surge como único diagnóstico de alterações mentais no pós-parto (Junior, 2017).

O puerpério para algumas mulheres pode se tornar um período desgastante, isto porque além de passar por diversas mudanças hormonais, esta mulher enfrentará uma nova função em sua vida, o papel materno, que pode se tornar assustador e comprometer a relação mãe e bebê. Porém todo este carrossel de emoções é natural e esperado dentro deste quadro perinatal, o que dever ser observado é a exacerbação dessa sintomatologia, que pode evoluir para algo patológico. O conhecimento por parte dos profissionais de saúde, dos sinais e sintomas associados às doenças psicológicas puerperais, a diferenciação do fisiológico e do patológico, a ciência que existem outras doenças mentais decorrentes da vulnerabilidade psicológicas destas mulheres e não somete a depressão pós-parto, é essencial para desenvolver uma assistência adequada, um tratamento eficaz e evitar maiores danos à gestante ou puérpera e ao seu bebê. (Viana et. al, 2020).

Segundo o Manual Técnico do Ministério da Saúde: Atenção Humanizada ao Recém- Nascido- Método Canguru (2016), a depressão pós parto pode atingir cerca de 15\% das puérperas, seus sintomas desenvolvem-se de forma tardia. Incluem: sinais de tristeza, cansaço, irritabilidade sentimentos de solidão e falta de interesse em realizar atividades diárias com o bebê. As puérperas apresentam um comportamento mecânico e operatório no qual as trocas com a criança se mostram pobres, sem expressões de afeto e as interações lúdicas são quase inexistentes. Não conseguindo ajustar sua linguagem à da criança, a mãe a priva de estímulos e informações, o que traz prejuízos cognitivos e emocionais para o recém-nascido.

O baby Blues é um transtorno mental no qual apresenta os mesmos sintomas que a DPP mas diferencia-se em intensidade e duração. Ele ocorre de forma imediata e pode regredir a maneira que a mãe vai se adaptando a nova rotina de cuidados com o bebê. Por apresentarem semelhanças e poucos estudos na área, é comum que haja equívoco no diagnóstico destas patologias, gerando falhas no cuidado com a puérpera.

O transtorno de estresse pós-traumático (TEPT) também é comum neste contexto e pode acontecer em consequência de intercorrências durante o parto, violência obstétrica ou complicações neonatais no qual a mulher experimenta sentimentos 
de angustia, dor, medo intenso e perda de controle. Após viver um parto traumático, algumas mulheres passam a apresentar, no pós-parto, recordações aflitivas do parto, por meio de imagens, ideias, sonhos ou emoções, e desenvolvem fugas de situações, pessoas, lugares e pensamentos que a remetam para o momento do parto. Associado a esse quadro, elas também apresentam hiperexcitabilidade e entorpecimento afetivo. Esses sintomas podem se agravar quando não tratados, podendo levar esta mulher a um quadro de Psicose puerperal, uma condição na qual apresenta grave comprometimento emocional e psíquico com necessidade de intervenção psiquiátrica (Cantilino et. al, 2010).

Assim, é importante ressaltar que, a identificação precoce dos sinais apresentados pela mulher desde o pré-natal é fundamental e vai direcionar os cuidados que serão prestados pelo Enfermeiro, por este motivo é importante que o profissional esteja atento as relações familiares, contexto social e função psíquica desta mulher, a atendendo com atenção e respeito, não desvalorizando suas tristezas e dores, para que não haja agravos na saúde.

\subsection{Papel do enfermeiro com pacientes com transtornos mentais puerperais - o início de uma longa jornada}

No que diz respeito ao papel do enfermeiro no contexto transtornos mentais puerperais, observou-se que, em relação à depressão pós-parto, a maioria dos profissionais de enfermagem não tem uma definição formada sobre esta doença em particular, nem sobre outros transtornos mentais que possam aflorar no puerpério, e assim, não conseguem correlacioná-la com fatores que possam gerar maiores agravos à puérpera. Mas, de acordo com a análise realizada, eles se consideram capazes para identificar uma possível patologia a partir de diversas características apresentadas pela mulher, como: desinteresse pelo bebê e pela realização dos cuidados que este necessita, desânimo ao falar do recém-nascido, apatia, entre outros (Camilo, 2016).

Esta identificação de prováveis patologias mentais puerperais tem sua origem durante o pré-natal, visto que grande parte dos enfermeiros tem a assistência no pré-natal como um dos cuidados mais importantes a ser prestado às gestantes. É considerado um momento para estabelecer empatia com a paciente, criar laços, oferecer acolhimento e deste modo possibilitar a identificação de alguma sintomatologia psicopatológica. Assim, uma estratégia efetiva encontrada nos artigos analisados, é a realização de grupos de gestantes, que procura atender as necessidades educativas, proporcionando espaços favoráveis para as trocas de ideias, expectativas entre gestantes, com compartilhamento de experiências das que já passaram por depressão pósparto, familiares e profissionais da saúde (Viana et.al, 2020).

Além disso, como as consultas no pré-natal, as do pós-natal e a visita domiciliar (VD) puerperal realizada pelas equipes de atenção primária à saúde aparece nos artigos analisados como uma das intervenções do enfermeiro, que abre espaço para o acolhimento à mulher e ao seu bebê, proporcionando-lhe educação e promoção em saúde. (Silva et.al, 2020). A visita domiciliar pode-se mostrar um importante momento para estas equipes detectarem mudanças físicas, comportamentais e emocionais precocemente, desenvolver ações educativas, identificar riscos e vulnerabilidades da família, estabelecer vínculo profissional-usuário e potencializar a proteção à saúde da mulher e da criança no pós-parto. (Baratieri \& Natal, 2018).

Este aspecto se conecta com uma estratégia que ganhou relevância entre os artigos da amostra, que foi a importância dada pela enfermagem ao agir em equipe, estabelecer vínculos, realizar uma abordagem familiar para que haja uma resposta positiva na prevenção e tratamento da depressão pós-parto e de outros transtornos mentais puerperais. Este fato foi mais evidente em estudos sobre a ação dos enfermeiros que trabalham em saúde pública. De acordo com a análise feita, estes profissionais se mostram com melhor preparo para identificar e tratar mulheres com doenças mentais puerperais e assim, encaminhá-las quando necessário para unidades especializadas (Souza et.al, 2018).

Seguindo a linha de raciocínio dos fatos apresentados, podemos afirmar que o enfermeiro que trabalha nas unidades de saúde, tem aptidão para trabalhar com a saúde mental das gestantes e puérperas e deve estar incluído no seu plano de cuidados, a detecção de um possível quadro de depressão puerperal ou outra psicopatologia decorrente do pós-parto. Uma vez que, em consonância com o Conselho Federal de Enfermagem (Resolução COFEN No 524/2016), compete ao enfermeiro 
garantir a integralidade do cuidado à mulher e ao recém-nascido, inseparável das questões físicas, emocionais, de seu contexto familiar, comunitário e de relações sociais desde o pré-natal até ao puerpério, por meio da consulta de enfermagem (Brasil, 2016).

Pensa-se, ainda, que o acompanhamento permanente nas consultas de pré-natal e nas visitas domiciliares, favoreça a criação de laços entre os profissionais as gestantes e suas famílias, facilitando a construção de uma anamnese mais fidedigna, a observação mais realista da rotina das gestantes e puérperas, das suas relações familiares e sociais, facilitando assim a identificação de alguma vulnerabilidade, de algum predisponente ou sintomatologia que possa ser sugestiva de transtorno mental puerperal ou de risco de desenvolvimento. Diversos estudos confirmam a importância do conhecimento dos fatores de risco e de proteção da depressão pós-parto para o planejamento e execução de ações preventivas ainda na fase pré-natal, para viabilizar a promoção do cuidado integral à gestante e puérpera (Arrais et. al, 2018).

No entanto a ausência de um conhecimento específico que visa acoplar práticas e estratégias para cuidar desta problemática, dificultam a percepção imediata de algum comportamento indicador de transtorno mental puerperal e quando este é precocemente detectado não há uma conduta protocolada pela instituição, ou embasada empiricamente, o que prejudica a definição de um diagnóstico correto da patologia e, por consequência, a realização do devido atendimento. (Costa et. al, 2018). Neste âmbito, torna a conduta assistencial de enfermagem, frágil e ineficiente, resultando em casos subdiagnosticados. Com isto, a patologia pode se exacerbar acometendo ainda mais a saúde da mãe e, por conseguinte, a do bebê (Maciel et. al, 2019).

A falta de estudos científicos sobre esta tônica, a ausência de estímulos à sua abordagem com as gestantes e seus familiares nas unidades de saúde e a inexistência de um aprofundamento sobre a saúde mental puerperal na disciplina de saúde da mulher a nível acadêmico ou como uma especialidade de pós-graduação, também pode estar entre um dos agentes facilitadores da banalização da doença e para o seu anonimato entre muitos dos profissionais de enfermagem. Juntamente com estes aspectos podemos incluir a sobrecarga de trabalho dos enfermeiros, presente em algumas literaturas, como um dos obstáculos para o aprofundamento sobre a saúde mental durante a gravidez e no pós-parto (sinais e sintomas, fatores predisponentes, entre outros), para se dedicarem mais às informações explicitas e implícitas (movimentos corporais, silêncio) apresentadas pela grávida ou puérpera e sua família, bem como para o desenvolvimento de estratégias que possam enfraquecer o surgimento de alguma psicopatologia puerperal (Bitti et. al, 2018).

Tal como os enfermeiros da atenção básica, os enfermeiros da área de obstetrícia, conforme os artigos analisados da amostra, também se deparam com limitações no enfretamento dos transtornos puerperais, apesar de possuírem um maior desafio de adequação técnica do modelo de identificação e prevenção da depressão pós-parto e de outros transtornos mentais advindos do pós-parto, com investimento em métodos de conforto e cuidados, bem como o uso adequado de tecnologia disponível e desejável, afim de minimizar as consequências decorrentes do parto e do pós-parto, uma vez que podem ser determinantes para o desencadear de algum tipo de transtorno mental puerperal. Assim, neste cenário o papel do enfermeiro foi muito associado a apoio emocional, com oferta de palavras de afeto e de conforto tanto à gestante, quanto à família, afim de tranquiliza-los, diminuir a angústia e a ansiedade (Aloise, Ferreira \& Lima, 2017).

Supõe-se que o escasso tempo de relacionamento entre profissional e paciente seja um aspecto limitante para criar laços e dificultar, assim, a detecção por parte do enfermeiro obstetra, de uma sintomatologia psicopatológica. Associado a este aspecto temos também a falta de preparo e conhecimento destes profissionais sobre as doenças mentais puerperais e de seus fatores desencadeantes. Muitos estudos apontam como fatores de risco de desenvolvimento de transtornos mentais puerperais os problemas decorrentes do momento do parto, ausência do companheiro e/ou da família mais próxima na hora do parto (Bitti et. al, 2018).

Deste modo, a incorporação de uma equipe multiprofissional, da qual façam parte profissionais da área de saúde mental, médicos, enfermeiros, técnicos de enfermagem, assistentes sociais, entre outros, nas equipes de saúde de hospitais 
obstétricos, poderia ser uma mais valia na identificação e manejo de casos de transtornos mentais ou com risco de evolução no pós-natal. Além disso, deve-se considerar a necessidade de implantar ações voltadas para o acompanhamento da gestante no momento do parto, visando treinar as equipes de saúde, especialmente os enfermeiros, a fim de capacitá-los para oferecerem à mulher um suporte global (biológico, psicológico e social) e humanizado de que necessita nesse momento tão delicado, sabendo que este apoio pode ser benéfico para a redução do risco de depressão puerperal e outras comorbidades mentais no futuro. (Hartmann et. al, 2017).

Ou seja, embora o Ministério da Saúde disponibilize manuais descriminando a atuação do enfermeiro em relação aos aspectos emocionais da gravidez e do puerpério, esta é superficial e a nível conceitual, sem destacar uma conduta em caso de suspeita de alteração psicológico e muito menos referenciando um cuidado assistencial onde a saúde mental é avaliada holisticamente. Limita-se a um foco meramente informativo e educacional sobre as diferentes etapas da gravidez, as alterações físicas inerentes a estas, e sobre os cuidados com o bebê (Brasil, 2006).

Também se observa por parte deste órgão uma preocupação pela amplitude e integralidade do cuidado à mulher durante o pré e o pós-natal, com estratégias humanizadas, como por exemplo, com a criação de alguns programas específicos como é o caso da "Rede Cegonha", existente em alguns municípios, que estende a assistência ao binômio mãe-bebê até aos dois anos de idade deste, o "Método Canguru", que visa estreitar o vínculo mãe-filho pelo contato pele-a-pele, especialmente no ambiente neonatal, eles se destinam à garantia do cuidado ao bebê e de sua adaptação à nova realidade, eles não incorporam programas que abordam a assistência à mulher de forma global. Apesar do Método Canguru ajudar a diminuir a ansiedade e a angustia materna e surja nas literaturas como um atenuante para o desenvolvimento de transtornos mentais no pós-parto (Brasil, 2017).

É necessário também, que o setor da saúde e os seus profissionais estejam abertos para as mudanças sociais que ocorrem e cumpram de maneira mais ampla o seu papel de educadores e promotores da saúde. As gestantes constituem o foco principal do processo de aprendizagem, porém a enfermagem não pode deixar de atuar, com os seus companheiros e familiares (Brasil, 2006). A posição do homem na sociedade está mudando e algumas literaturas já ressaltam que transtornos puerperais, como a depressão pós-parto, não é exclusiva das mulheres, muitos pais já foram diagnósticos com a doença e que igualmente à depressão pós-parto materna, influência a saúde e desenvolvimento do bebê. Portanto, os estes profissionais devem promover o envolvimento dos homens no contexto gestacional e puerperal (Caparrós-Gonzáleza \& Rodriguez-Muñoz, 2020).

\subsection{Estratégias e protocolos na assistência às puérperas com transtornos mentais puerperais}

Dos estudos analisados neste trabalho se mostraram muito vagos em estratégias, protocolos de assistência ou mecanismos de rastreio, identificação de sinais e sintomas reveladores de transtornos mentais puerperais. A aplicação da escala de Edimburgo - Edinburgh Postnatal Depression Scale (EPDS) - que é uma escala utilizada para medir a sintomatologia depressiva, foi a única escala de rastreamento referenciada. Segundo os estudos ela se mostrou fidedigna em relação aos resultados evidenciados (Alvarenga et. al, 2017).

Também foi considerada simples, rápida de realizar e autoaplicável, sendo a sua disseminação na área da saúde sugerida, uma vez que pode ser ideal para uso na rotina clínica de profissionais que não são especializados na área da saúde mental e por não onerar os serviços especializados. O amplo uso da escala poderá assim, ser associado a um aumento nos índices de diagnóstico e tratamento da doença, minimizando assim seus possíveis efeitos nocivos sobre a mãe e o filho (Araujo et. al, 2019).

Alguns estudos realizados pelo Ministério da Saúde também vão ao encontro dos resultados encontrados pelos estudos da amostra e firmam a importância desta escala nas unidades de saúde pública e privada. Porém ressaltam uma afirmativa que pode justificar a aplicabilidade acanhada desta escala no Brasil, ao contrário de outros países, como Reino Unido, onde esta é 
utilizada rotineiramente com as puérperas, que é a carência de estudos comprobatórios sobre a consolidação de resultados favoráveis ao uso da escala de rastreamento de depressão pós-parto e de outros transtornos mentais pelo país, principalmente entre as capitais e entre populações de zona rural ou com baixos níveis de escolaridade ou não alfabetizadas (Brasil, 2013).

Além disso, por se tratar de uma escala de rastreamento, o diagnóstico só poderá ser confirmado por meio de instrumentos de diagnóstico clínico e por profissionais treinados. A falta de verba para a compra de equipamentos e para capacitar os profissionais, pode se mostrar também, um entrave para a utilização destes instrumentos, especialmente se tratando de regiões do Brasil mais carentes de recursos humanos especializados e financeiros (Brasil, 2013).

Outras literaturas também nos mostraram particularidades passíveis de reflexão no que concerne à fraca adesão ao uso de procedimentos de rastreamento, prevenção, tratamento dos transtornos mentais puerperais como, o conhecimento limitado e despreparo de alguns enfermeiros, em conjunto com a lacuna existente nas unidades de saúde em relação à abordagem da saúde mental no pré-natal. Estas, revelam um panorama ainda disseminado no país de que a "depressão pós-parto é doença de rico". Podendo, assim, serem predisponentes da insensibilidade apresentada por estes profissionais para enxergarem as necessidades das pacientes durante o quadro gestacional e puerperal, e da subestima dos seus relatos, seus sinais e sintomas, não conseguindo vislumbrar, por conseguinte, as escalas de rastreamento, como a EPDS, ou outros recursos, como instrumentos com aplicabilidade nas suas atividades diárias e com benefícios para a saúde das gestantes, puérperas e de seus bebês (Fiocruz, 2020).

Um outro dado que surgiu na nossa analise amostral, embora de forma muito discreta, foi a utilização da tecnologia a favor da prevenção da depressão pós-parto. O estudo internacional revelou que as mulheres perinatais dão preferência ao recebimento de informação e prevenção da depressão perinatal on-line: por e-mail ou por sites especializados. O interesse por um programa de prevenção da depressão pós-parto de forma individualizada, privada, onde o acesso à informação está disponível para ser acessado onde e quando for mais conveniente para estas, se expressou positivamente de forma unânime. $\mathrm{O}$ que levou os autores do estudo a exaltarem esforços para o desenvolvimento de programas e recursos no formato on-line para a prevenção e tratamento das psicopatologias puerperais, difundindo efetivamente, o uso global das tecnologias a fim de impactar as mulheres perinatais em todo o mundo (Osma et. al, 2019).

De acordo com pesquisadoras da FIOCRUZ (2020), a orientação on-line sobre psicopatologias no puerpério é uma estratégia muitas vezes recorrida aqui no Brasil. Porém o tema é abordado na internet, principalmente em blogs, com uma qualidade de informação variável. Onde a maioria das postagens se referem a encorajamento, empoderamento da mulher e sobre confiar no serviço de saúde. Deste modo, pensa-se que, programas de prevenção, rastreio on-line, como o mencionado no estudo da nossa amostra, seria uma alternativa bem aceite no país. Não apenas pela familiaridade do público alvo com o meio de apresentação do conteúdo, mas também por ser uma forma de acesso à informação, atualmente, acessível na maior parte do território brasileiro.

Além do mais, também é um recurso que permite manter a privacidade das gestantes e puérperas, garantido a confidencialidade dos dados registrados, sendo uma forma menos constrangedora para estas exporem as suas angústias, medos e sentimentos. Pois, as mesmas pesquisadoras também relataram em seus trabalhos que, por mais que o pré-natal no Brasil não aborde a saúde mental das mulheres, apenas metade das que manifestam sintomas depressivos procuram algum tipo de atendimento especializado. O estigma de que depressão pós-parto é "frescura", é "doença de rico" e do sentimento de ser considerada uma "mãe má e incapaz de cuidar de seu filho", é a uma barreira na busca de tratamento pelas gestantes e mães (Fiocruz, 2020). 


\section{Conclusão}

Com este estudo entendemos que a gestação é um período crítico do ciclo vital da mulher, que exige adaptações e reorganização psíquica, e que é imprescindível ao enfermeiro o domínio sobre a fisiologia da gravidez e como esta afeta a condição clínica da mulher para minimizar agravos futuros, tanto para a sua saúde, quanto para a do bebê. No entanto existe desconhecimento, por parte destes profissionais, em relação aos transtornos mentais puerperais e limitado número de pesquisas sobre esta temática, que amplifiquem o seu conhecimento e definam, assertivamente, a sua atuação.

Verificamos também que a depressão pós-parto é tema quase exclusivo de diversos estudos que abordam a questão saúde mental durante a gestação e o pós-parto, dando com isso a falsa ideia de que é única doença dentro das psicopatologias puerperais e que apenas ela é passível de acometer as grávidas e puérperas, o que consolida ainda mais a lacuna no aprendizado dos enfermeiros sobre este assunto.

Neste contexto, restrito à depressão pós-parto, a atuação do enfermeiro é ressaltada, principalmente a dos enfermeiros que trabalham no pré e pós-parto, onde a consulta de enfermagem é entendida como o momento crucial para a identificação de qualquer alteração psicopatológica sugestiva desta doença. No entanto, é também onde o fechamento correto do diagnóstico seguido de um encaminhamento adequado, esbarra nesse conhecimento deficitário da maioria dos profissionais, o que contribui para um avanço da doença e de um somatório de complicações decorrentes.

Assim, entende-se como uma necessidade urgente: a introdução da saúde mental nos programas nacionais de prénatal, capacitação dos enfermeiros na área de saúde mental puerperal, estimulo para a promoção da educação permanente, investimento em meios de reconhecimento, diferenciação e acompanhamento dos transtornos mentais puerperais, incitação à realização de pesquisas que busquem identificar fatores de risco e contribuam para o desenvolvimento de protocolos de cuidados assistenciais, sem deixar de lado a atenção humanizada, assim como escalas de rastreamento para identificação em tempo hábil de um possível quadro patológico. O que trará benefícios na detecção precoce das doenças mentais puerperais, assim como de outros embaraços que possam afetar o ciclo gravídico puerperal. Podendo, desta forma, repercutir positivamente na prevenção de problemas futuros na saúde da mãe e do bebê, na relação e estabelecimento de vínculos entre eles.

Apesar deste estudo apresentar como limitação o pouco tempo de pesquisa, o que pode ter influenciado o número reduzido de artigos e bibliografia encontrada sobre o assunto, particularmente em relação à atuação do enfermeiro, o que muitas vezes nos deixou em um círculo vicioso, com um discurso repetitivo, foi assimilado o quanto ainda existe para ser realizado neste âmbito e como isso é importante. A saúde mental perinatal é uma questão de saúde pública pelo seu alto impacto na vida da mulher e pelos efeitos nocivos que pode acarretar sobre a sua saúde a da criança e sobre as suas relações familiares.

A fim de contribuir mais para o conhecimento na área da saúde mental no período puerperal, apontamos sugestões de temas para trabalhos futuros como: "O impacto da pandemia nos transtornos mentais puerperais" e "Os impactos dos transtornos mentais puerperais ao recém-nato e a primeira infância” pois destacam-se como assuntos extremamente relevantes, atuais e pouco explorados.

\section{Referências}

Aloise, S. R. (2019). Depressão Pós-Parto: Identificação de Sinais, Sintomas e Fatores Associados em Maternidade de referência em Manaus. Revista Oficial do Conselho Federal de Enfermagem, Manaus, 5 (7), 40-45. http://revista.cofen.gov.br/index.php/enfermagem/article/view/2455/584.

Alvarenga, P. \& Frizzo, G. B. (2017). Stressful Life Events and Women's Mental Health During Pregnancy and Postpartum Period. Paidéia (Ribeirão Preto), Porto Alegre, 27 (66), 51-9. FapUNIFESP (SciELO). http://dx.doi.org/10.1590/1982-43272766201707. 
Araújo, I., Aquino, K., Fagundes, L. \& Santos, V. (2019). Postpartum Depression: epidemiological clinical profile of patients attended in a reference public maternity in salvador-ba. Revista Brasileira de Ginecologia e Obstetrícia / Rbgo Gynecology And Obstetrics. Rio de Janeiro, 41 (3), 155-63. http://dx.doi.org/10.1055/s-0038-1676861.

Arrais, A. R., Araujo, T. C, C. F. de \& Schiavo, R. A. (2018). Fatores de Risco e Proteção Associados à Depressão Pós-Parto no Pré-Natal Psicológico. Psicologia: Ciência e Profissão, São Paulo, 38 (4), 711-729, FapUNIFESP (SciELO). http://dx.doi.org/10.1590/1982-3703003342016.

Baratieri, T. \& Natal, S. (2019). Ações do programa de puerpério na atenção primária: uma revisão integrativa. Revista Ciência \& Saúde Coletiva. [S.L.]Florianópolis, 24 (11), 4227-4238. FapUNIFESP (SciELO). http://dx.doi.org/10.1590/1413-812320182411.28112017.

Bitti, V., Reis, L., Trindade, W., Emerick, L. \& Pereira, W. (2018). Atuação dos Enfermeiros na Prevenção e Acompanhamento da Depressão Puerperal. Revista Enciclopédia Biosfera, 15 (27), 1424-1436. Centro Cientifico Conhecer. http://dx.doi.org/10.18677/encibio_2018a122.

Brasil. Ministério da Saúde. (2006). Manual Técnico - Pré-Natal e Puerpério Atenção Qualidade e Humanização. http://bvsms.saude.gov.br/bvs/publicacoes/manual_pre_natal_puerperio_3ed.pdf.

Brasil. Ministério da Saúde. Avaliação da atenção ao pré-natal, ao parto e aos menores de um ano na Amazônia Legal e no Nordeste. (2010 a 2013). https://bvsms.saude.gov.br/bvs/publicacoes/livro_avaliacao_da_atencao_ao_pre-natal_web.pdf.

Brasil. Constituição. (2016). Resolução Cofen $n^{o} N^{o}$ 524/2016, de 27 de junho de 2016. http://www.cofen.gov.br/resolucao-cofen-no-05162016_41989.html.

Brasil. Ministério da Saúde. (2017). Atenção Humanizada ao Recém-Nascido Método Canguru Manual Técnico. https://bvsms.saude.gov.br/bvs/publicacoes/atencao_humanizada_metodo_canguru_manual_3ed.pdf.

Brasil. Ministério da saúde. (2000). Assistência pré-natal - Manual Técnico. https://bvsms.saude.gov.br/bvs/publicacoes/cd04_11.pdf.

Camilo, B. S. et. al. (2016). Ações de Educação em Saúde na Atenção Primária a Gestantes e Puérperas: Revisão Integrativa. Revista de Enfermagem Ufpe On-Line, Santa Maria, 10 (6), 4894-4901. https://periodicos.ufpe.br/revistas/revistaenfermagem/article/download/11270/12905.

Cantilino, A. et al. (2010). Transtornos psiquiátricos no pós-parto. Revista de Psiquiatria Clínica. Archives of Clinical Psychiatry, 37 (6), $288-294$. https://www.scielo.br/j/rpc/a/nfBndszPxgSTqkh9zXgpnjK/?lang=pt\#ModalArticles.

Caparrós-Gonzáleza, R. A. \& Rodriguez-Muñoz, M. F. (2020). Depresión Posparto Paterna: Visibilidad e Influencia en la Salud Infantil. Colegio Oficial de La Psicologia de Madrid, 161-163. https://journals.copmadrid.org/clysa/art/clysa2020a10.

Costa, D. O., Souza, F. I. S., Pedroso, G. C. \& Strufaldi, M. W. L. (2018). Transtornos mentais na gravidez e condições do recém-nascido: estudo longitudinal com gestantes assistidas na atenção básica. Revista Ciência \& Saúde Coletiva. [S.L.], 23 (3), 691-700, FapUNIFESP (SciELO). http://dx.doi.org/10.1590/1413-81232018233.27772015.

Ercole, F. F., Melo, L. S. \& Alcoforado, C. L. G. C. (2014). Revisão integrativa versus revisão sistemática. Revista Mineira de Enfermagem. http://reme.org.br/artigo/detalhes/904.

Fiocruz. Escola Nacional de Saúde Pública Sergio Arouca. (2020). Transtornos mentais durante a gravidez e pós-parto esbarram em estigma, mostram pesquisadoras. http://informe.ensp.fiocruz.br/noticias/48306.

Junior, A. R. F. et. al. (2017). O Enfermeiro no Pré-Natal de Alto Risco: Papel Profissional. Revista Baiana de Saúde Pública. Fortaleza, 41 (3), 650-667. https://rbsp.sesab.ba.gov.br/index.php/rbsp/article/view/2524.

Hartmann, J. M., Mendoza-Sassi, R. A. \& Cesar, J. A. (2017). Depressão entre puérperas: prevalência e fatores associados. Cadernos de Saúde Pública, Rio Grande, 33 (9), 1-15. FapUNIFESP (SciELO). http://dx.doi.org/10.1590/0102-311x00094016.

Macedo, A. F. \& Pereira, A. T. (2014). Saúde Mental Perinatal. https://www.wook.pt/livro/saude-mental-perinatal-ana-telma-pereira/15746722.

Montenegro, C. A. B. \& Filho, J. R. (2017). Rezende Obstetrícia Fundamental. Guanabara Koogan. https://docero.com.br/doc/1cn551.

Maciel, L. P. et. al (2019). Transtorno mental no puerpério: riscos e mecanismos de enfrentamento para a promoção da saúde. Revista Fun Care On-line. 11 (4), 1096-1102. http://dx.doi.org/10.9789/2175-5361.2019.v11i4.1096-1102.

Organização Mundial de Saúde. (2010). MI-GAP Manual de Intervenções Programa de Ação Mundial em Saúde Mental MI-GAP para transtornos mentais, neurológicos e por uso de álcool e outras drogas na rede de atenção básica à saúde. Versão 1.0. Programa de Ação Mundial em Saúde Mental. https://www.who.int/mental_health/publications/IG_portuguese.pdf.

Organização Pan-Americana de Saúde. (2017). Depressão é tema de campanha da OMS para o Dia Mundial da Saúde de 2017. https://www.paho.org/bra/index.php?option=com_content\&view=article\&id=5321:depressao-e-tema-de-campanha-da-oms-para-o-dia-mundial-da-saude-de$2017 \&$ Itemid $=839$.

Osma, J., Suso, R. C., Martínez, B. V. \& Barrera, A. Z. (2019). Content and format preferences of a depression prevention program: a study in perinatal women. Servicio de Publicaciones de la Universidad de Murcia. Anales de Psicología, Palo Alto, 36 (1), 56-63. http://dx.doi.org/10.6018/analesps.356051.

Silva, J. F. et al. (2020). Intervenções do enfermeiro na atenção e prevenção da depressão puerperal. Revista de enfermagem UFPE On Line. https://doi.org/10.5205/1981-8963.2020.245024.

Viana, M. D. Z. S., Fettermann, F. A. \& Cesar, M. B. N. (2020). Estratégias de enfermagem na prevenção da depressão pós-parto. Revista de Pesquisa Cuidado é Fundamental Online, 12, 953-957. http://dx.doi.org/0.9789/2175-5361.rpcfo.v12.6981. 